ISSN 1392-3196 / e-ISSN 2335-8947

Zemdirbyste-Agriculture, vol. 102, No. 4 (2015), p. 457-464

DOI 10.13080/z-a.2015.102.059

\title{
Prioritization of feasible physiological parameters in drought tolerance evaluation in sorghum: a grey relational analysis
} \author{
Ruidong HUANG ${ }^{1}$ \\ ${ }^{1}$ Agronomy College, Shenyang Agricultural University \\ Shenyang, Liaoning Province 110866, People's Republic of China \\ E-mail: zhouyufei2002@aliyun.com; r_huang@126.com \\ ${ }^{2}$ Agro-environmental Protection and Supervision Center \\ Shenyang, Liaoning Province 110034, People's Republic of China \\ ${ }^{3}$ Department of Crop and Soil Sciences, University of Georgia \\ 1109 Experiment str., Griffin, GA 30223, USA
}

Na WANG ${ }^{1}$, Yitao WANG ${ }^{2}$, Jialin $\mathrm{YU}^{3}$, Yufei ZHOU${ }^{1}$, Qi WU ${ }^{1}$, Yue GAO ${ }^{1}$, Wenjuan XU ${ }^{1}$,

\begin{abstract}
Identification and evaluation of drought tolerant germplasm is the primary step for sorghum (Sorghum bicolor L. Moench) breeding and utilization under drought conditions. The objective of this study was to use a grey relational analysis to investigate the role of feasible physiological parameters in evaluating drought tolerance in sorghum. Four sorghum varieties were cultivated in pots with two water treatments, including normal watering (75-80\% of the soil moisture capacity) and water deficit (45-50\% of the soil moisture capacity), which occurred at jointing stage, anthesis and filling stage, respectively. Drought tolerance index of yield was used as the key indicator to evaluate sorghum performance under drought. The grey relational degree of the investigated parameters decreased in the order of transpiration rate, stomatal conductance, photosynthetic rate, soluble sugar content, proline content, relative water content, activity of catalase, activity of superoxide dismutase and activity of peroxidase, implying that drought tolerance for guaranteeing sorghum yield formation was the most related to gas exchange parameters. Water content was a very sensitive parameter of plant growth under drought stress and was more important as compared to the activities of antioxidant enzymes. Results of this research suggested that feasible physiological parameters could be used in the evaluation of drought tolerance to improve the efficiency and accuracy of selection.
\end{abstract}

Key words: analytic technique, physiological traits, Sorghum bicolor, water deficit.

\section{Introduction}

Sorghum is mostly cultivated in arid and semiarid regions of Africa and Asia. For instance, the harvested area of sorghum in Africa and Asia accounted for $81 \%$ of the world according to 2013 data (FAO, 2015). Moreover, sorghum continues to be an important crop with multiple applications for food, brewing, forage, and biofuel throughout the world. Although sorghum is considered as a drought-tolerant crop (Shan, $\mathrm{Xu}, 2009$ ), its production often suffers from drought in those regions. Sorghum breeders seek to utilize drought tolerant germplasm to mitigate drought stress risk to sorghum production (Borrell et al., 2014). During the sorghum breeding process, it is crucial to improve the screening efficiency and identifying accuracy.

Drought stress can notably impact various physiological and biochemical processes in sorghum plants, such as photosynthetic characters (Ogbaga et al., 2014; Zhou et al., 2014) and osmotic adjustment (Zhou et al., 2013), etc., which result in an obvious decline in final productivity. Lu et al. (2011) stated that adaptive responses of crops induced by drought included morphological changes and biochemical and physiological reactions. Further, drought tolerance might be dominated by complicated multi-genetic systems. In previous reports, various physiological parameters were used to identify drought tolerant germplasm and crop behaviour under drought conditions (Ober et al., 2005; Lu et al., 2011; Chen et al., 2012; Assefa et al., 2013). Although many indicators of drought tolerance were selected in these studies, the selection criterion was not completely identical except for the yield as the primary trait in evaluating the drought tolerance of crops. Up to now, which physiological parameter plays the most important role in evaluating the drought tolerance of sorghum has not been well understood. Hence, it is necessary to use certain simple and practicable physiological parameters to evaluate drought tolerance especially when the identifying materials are numerous. A more direct and effective method for evaluating traits and 
guiding the breeding of drought tolerant crop is needed urgently.

Given that crop behaviour (such as drought tolerance) could be associated with multiple factors and be treated as a complicated system, the method of grey relational analysis (GRA) can be used to identify the primary factors that contribute to the drought tolerance. The GRA was initially applied by Deng (1989), and has been proved to be an effective way to explain the correlation between system behaviour (such as crop production) and relevant affecting factors (Li et al., 2007; Jia, Shao, 2013; Wang et al., 2013). Since the factors have different effects on crop behaviour, they can be assigned with different values to reflect their importance. Wang et al. (2013) analyzed the habitat factors to discriminate which factor was closely associated with winter wheat freeze injury by using the method of GRA. However, little is known about the application of the method in evaluating the drought tolerance of sorghum.

The objective of this study was to provide a grey relational analysis (GRA) to explore the prioritization of feasible physiological parameters in evaluating drought tolerance in sorghum. The results possess the potential to promote identifying accuracy and guide sorghum breeding practices under drought conditions.

\section{Materials and methods}

Site and soil. The research was carried out form 2010-2012 in the experimental base of Shenyang Agricultural University, China $\left(41^{\circ} 49^{\prime} \mathrm{N}, 123^{\circ} 34^{\prime} \mathrm{E}\right)$. Its altitude is $25 \mathrm{~m}$ from the sea level. The average annual rainfall and mean monthly temperature are $500 \mathrm{~mm}$ and $8.3^{\circ} \mathrm{C}$. The soil of the experiment was collected from the top surface layer $(0-20 \mathrm{~cm})$ of arable soil near the experimental base of Shenyang Agricultural University. Soil was silt loam with a $\mathrm{pH}$ of 7.0, organic matter content of $30.82 \mathrm{~g} \mathrm{~kg}^{-1}$, alkali hydrolysable $\mathrm{N}$ of $104.58 \mathrm{mg} \mathrm{kg}^{-1}$, available $\mathrm{P}$ of $78.33 \mathrm{mg} \mathrm{kg}^{-1}$, available $\mathrm{K}$ of $88.33 \mathrm{mg} \mathrm{kg}^{-1}$.

Experimental design and treatments. Sorghum (Sorghum bicolor L. Moench) four varieties 'Jiza305', 'Jiza127', 'Jinza106' and 'Jinza103' with different drought tolerance were selected from 31 sorghum varieties which were mainly cultivated in northeastern China. Five sorghum seeds were planted in plastic pots $(28 \mathrm{~cm}$ high and $33 \mathrm{~cm}$ diameter) filled with $20 \mathrm{~kg}$ soil and the seedlings were thinned to a single plant in each pot when plants reached third leaf stage. Diammonium phosphate $(2.5 \mathrm{~g})$ was applied in each pot as basal fertilizer and urea (3.3 g) was applied at jointing stage based on local sorghum cultivation. The experiment was established as a completely randomized design with three replications. Sorghum was planted on 17 May and harvested on 25 September in each year.

Sorghum plants were subjected to water stress by withholding irrigation to obtain $45-50 \%$ of the soil moisture capacity during the jointing stage (initiated on 26 June), anthesis (initiated on 23 July) and filling stage (initiated on 6 August), respectively. Control pots were well drained and regularly watered to keep $75-80 \%$ of the soil moisture capacity throughout the experiment. The soil moisture content was maintained by using soil moisture sensor HH2/ML2X ("Delta-T", UK). The drought stress period lasted for 7 days during the jointing stage, anthesis, and filling stage, respectively. A mobile rain shelter was used to protect the pots from the rain during the stress period. Physiological parameters were examined timely for different plants after each drought stress period. The drought-stressed plants were rewatered normally after each stress till mature.

A mobile photosynthetic system Li-6400 ("LiCor", USA) was employed to measure photosynthetic rate, stomatal conductance, and transpiration rate on the tenth leaf at jointing stage and on the second leaf from the top at anthesis and filling stage (15 replications per treatment) between 09:00 and 11:00 a.m. The selected leaves for the investigation were full-developed and healthy. The tenth leaf at jointing stage and the second leaf from the top at anthesis and filling stage were sampled and the relative water content (RWC) was determined by the following formula:

$$
\operatorname{RWC}(\%)=\frac{W_{f}-W_{d}}{W_{t}-W_{d}} \times 100 \text {, where } W_{f} \text { is the }
$$

fresh weight of leaf, $W_{d}$ - the dry weight of leaf, and $W_{t}$ - the turgid weight of leaf after being soaked in the deionized water for $24 \mathrm{~h}$ in the dark. Fresh leaves $(0.5 \mathrm{~g})$ were homogenized in $5 \mathrm{ml}$ of $0.1 \mathrm{M}$ phosphate buffer ( $\mathrm{pH} 7.8)$. The homogenate was centrifuged at $13.000 \mathrm{~g}$ for $20 \mathrm{~min}$ at $4^{\circ} \mathrm{C}$. Supernatant was used for the determining the activities of antioxidant enzymes as the crude extract for superoxide dismutase (SOD), catalase (CAT) and peroxidase (POD). The SOD, CAT and POD were quantified using a spectrophotometer U1900 ("Hitachi", Japan) according to the procedure described by Zhang (1992). Proline was extracted from fresh leaves $(0.5 \mathrm{~g})$ with $3 \%$ sulphosalicylic acid and measured by ninhydrin method according to Zhang (1992). Soluble sugar was determined by anthrone method described by Li (2000). At the mature stage, 15 plants per replicate were harvested and quantified to measure yield.

Analytical methods. Drought tolerance index of yield (DTIY) was used to estimate the comprehensive behaviour of all sorghum varieties under drought stress. DTIY was an integrated evaluation for crop drought tolerance (Lan et al., 1990). Specifically, DTIY was calculated as:

$$
\text { DTIY }=\frac{Y_{d}}{Y_{w}} \times \frac{Y_{d}}{Y_{a}} \text {, where } Y_{d} \text { is yield under }
$$

drought stress, $Y_{w}-$ yield under normal watering treatment, and $Y_{a}$ - average yield of all varieties under drought stress.

A grey relational analysis (GRA) was used to estimate the effect of the investigated parameters and identify the principal parameters in evaluating drought tolerance in sorghum. The method of GRA was initially applied by Deng (1989), and has been proved to be an effective way to explain the correlation between system behaviour (such as crop production) and relevant affecting factors (Li et al., 2007; Jia, Shao, 2013; Wang et al., 2013). 
According to GRA theory, the DTIY was characterized as the reference sequence to reflect drought tolerance and the relevant physiological parameters affecting drought tolerant behaviour were as the compared sequences. The specific calculation steps were expressed as follows:

The reference sequence can be defined as:

$X_{0}(k)=\left\{x_{0}(1), x_{0}(2), \ldots, x_{0}(n)\right\}$

signify the related $\mathrm{m}$ sequences to be compared as:

$X_{m}(k)=\left\{x_{m}(1), x_{m}(2), \ldots, x_{m}(n)\right\}$

Normalize the sequences by standard processing to ensure them to be comparable. The normalized reference sequence and comparing sequence can be expressed respectively as:

$$
\begin{aligned}
& y_{0}(k)=\left\{y_{0}(1), y_{0}(2), \ldots, y_{0}(n)\right\} \\
& y_{i}(k)=\left\{y_{i}(1), y_{i}(2), \ldots, y_{i}(n)\right\} \\
& I=1,2,3 \ldots m ; k=1,2,3 \ldots n .
\end{aligned}
$$

The grey rational coefficient $\left(L_{i}(k)\right)$ for the reference sequence and the comparing sequence was calculated as:

$$
L_{i}(k)=\frac{\min _{i} \min _{k}\left|y_{0}(k)-y_{i}(k)+\rho \max _{i} \max _{k}\right| y_{0}(k)-y_{i}(k) \mid}{\left|y_{0}(k)-y_{i}(k)\right|+\rho \max _{i} \max _{k} y_{0}(k)-y_{i}(k) \mid}
$$

where $\rho$ is distinguishing coefficient which ranges from 0 to 1 , typically assigned to 0.5 . Then, the grey relational degree was deduced form $r_{i}=\frac{1}{n} \sum_{k=1}^{n} L_{i}(k)$

The grey relational degree, ranging from 0 to 1 , indicated the effects of investigated factors. Higher grey correlation degree shows that the parameter has closer relationship with drought tolerance and the relational degree also expresses the importance of the parameter. Significance of main effects was determined using oneway analysis of variance in software SPSS 18.0. Means were separated by Duncan's multiple range test at $P=0.05$. Results were presented as the mean \pm standard deviation (SD).

\section{Results and discussion}

Drought inhibited the formation of sorghum yield. The greatest decline of yield induced by drought occurred at filling stage and the yield of the four sorghum varieties decreased by $25-44 \%$ (Table 1). Although drought at different stage can lead to the decline of yield, filling stage is the most critical period for yield formation and needs more water to maintain normal metabolism compared to the early growth stage. The varieties with higher DTIY had lesser yield reduction than those of lower DTIY at the same drought stressed stage. Yield is the primary criteria for the evaluation of drought tolerance in crops under drought conditions (Lu et al., 2011). Moreover, superior morphological, physiological and biochemical traits were also selected for evaluating drought tolerance of crops (Chen et al., 2012). For example, sorghum with stay-green trait expressed drought tolerance under drought conditions (Borrell et al., 2014). It was noted that these traits could eventually contribute to final yield particularly under drought conditions. Drought tolerant sorghum is generally considered to have relative higher yield under drought conditions (Shan, $\mathrm{Xu}, 2009$ ). The DTIY was used for evaluating drought tolerance of sorghum in this investigation. The index gave consideration to the interaction between genotypes and environments (drought), and the yield potential among the tested varieties under drought conditions. The variation of DTIY in different stressed stage suggested that the sensitivity of sorghum yield to drought that occurred at different growth stages differed significantly.

Table 1. Sorghum yield and relevant drought tolerance index of yield under drought stress occurred at different growth stages

\begin{tabular}{ccccc|ccc}
\hline & \multicolumn{4}{c}{} & \multicolumn{3}{c}{ Drought tolerance index of yield } \\
\cline { 2 - 8 } Variety & normal watering & $\mathrm{WD}_{\mathrm{j}}$ & $\mathrm{WD}_{\mathrm{a}}$ & $\mathrm{WD}_{\mathrm{f}}$ & $\mathrm{WD}_{\mathrm{j}}$ & $\mathrm{WD}_{\mathrm{a}}$ & $\mathrm{WD}_{\mathrm{f}}$ \\
\hline Jiza305 & $63.74 \pm 1.11 \mathrm{a}$ & $61.35 \pm 3.77 \mathrm{a}$ & $52.99 \pm 4.19 \mathrm{bc}$ & $47.85 \pm 1.71 \mathrm{de}$ & $1.09 \pm 0.12 \mathrm{a}$ & $0.82 \pm 0.11 \mathrm{~b}$ & $0.67 \pm 0.07 \mathrm{c}$ \\
Jinza106 & $65.15 \pm 1.33 \mathrm{a}$ & $56.06 \pm 5.03 \mathrm{~b}$ & $53.47 \pm 2.88 \mathrm{~b}$ & $46.87 \pm 1.19 \mathrm{de}$ & $0.93 \pm 0.12 \mathrm{~b}$ & $0.84 \pm 0.05 \mathrm{~b}$ & $0.65 \pm 0.03 \mathrm{c}$ \\
Jinza103 & $52.95 \pm 3.22 \mathrm{bc}$ & $45.18 \pm 3.01 \mathrm{de}$ & $43.55 \pm 1.57 \mathrm{ef}$ & $34.80 \pm 8.48 \mathrm{~g}$ & $0.95 \pm 0.19 \mathrm{ab}$ & $0.88 \pm 0.10 \mathrm{~b}$ & $0.58 \pm 0.23 \mathrm{~cd}$ \\
Jiza127 & $62.88 \pm 3.31 \mathrm{a}$ & $48.79 \pm 4.85 \mathrm{~cd}$ & $40.84 \pm 1.45 \mathrm{f}$ & $35.17 \pm 1.47 \mathrm{~g}$ & $0.91 \pm 0.10 \mathrm{~b}$ & $0.64 \pm 0.05 \mathrm{c}$ & $0.47 \pm 0.02 \mathrm{~d}$ \\
\hline
\end{tabular}

Note. $\mathrm{WD}_{\mathrm{j}}$ - water deficit at jointing stage, $\mathrm{WD}_{\mathrm{a}}$ - water deficit at anthesis, $\mathrm{WD}_{\mathrm{f}}$ - water deficit at filling stage; mean $\pm \mathrm{SD}$ followed by different letters are significantly different by the Duncan's $(p<0.05)$.

Results suggested that drought tolerance was the result of comprehensive physiological processes, such as photosynthesis, osmotic adjustment, antioxidant metabolism, which differed significantly between sorghum varieties under drought conditions (Tables 2-4). However, it was not obvious which physiological parameters played leading role in the difference of drought tolerance in sorghum. The summary of the investigated parameters of different physiological processes suggested that an explicit conclusion could not be achieved from the data, which was the assembly from different physiological reaction mechanism. GRA in this study offered a method to address this problem. It had been successfully used in evaluating crop performance with uncertain affecting parameters (Wang et al., 2013). Some environmental factors or agronomic traits were evaluated by GRA in maize and wheat under adverse conditions (Hu et al., 2012; Wang et al., 2013). 
Table 2. Variations of photosynthetic parameters of sorghum varieties under drought at jointing stage, anthesis and filling stage, respectively

\begin{tabular}{|c|c|c|c|c|c|}
\hline Stage & Variety & Treatment & $\begin{array}{l}\text { Photosynthetic rate } \\
\mu \mu \mathrm{mol} \mathrm{CO}_{2} \mathrm{~m}^{-2} \mathrm{~s}^{-1}\end{array}$ & $\begin{array}{l}\text { Stomatal conductance } \\
\text { mol } \mathrm{H}_{2} \mathrm{O} \mathrm{m}^{-2} \mathrm{~s}^{-1}\end{array}$ & 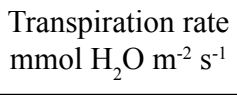 \\
\hline \multirow{8}{*}{ Jointing stage } & \multirow{2}{*}{ Jiza305 } & NW & $26.89 \pm 1.27 \mathrm{a}$ & $0.16 \pm 0.01 \mathrm{a}$ & $2.68 \pm 0.42 \mathrm{ab}$ \\
\hline & & WD & $21.98 \pm 0.47 \mathrm{~b}$ & $0.14 \pm 0.01 \mathrm{bc}$ & $2.2 \pm 0.04 \mathrm{bcd}$ \\
\hline & \multirow{2}{*}{ Jinza106 } & NW & $25.64 \pm 1.04 \mathrm{a}$ & $0.15 \pm 0.01 \mathrm{ab}$ & $2.69 \pm 0.17 \mathrm{ab}$ \\
\hline & & WD & $21.00 \pm 0.79 b$ & $0.13 \pm 0.02 \mathrm{c}$ & $2.27 \pm 0.07 \mathrm{bcd}$ \\
\hline & \multirow{2}{*}{ Jinza103 } & NW & $26.27 \pm 1.44 \mathrm{a}$ & $0.16 \pm 0.01 \mathrm{a}$ & $2.58 \pm 0.31 \mathrm{abc}$ \\
\hline & & WD & $20.60 \pm 1.13 b$ & $0.13 \pm 0.02 \mathrm{c}$ & $2.08 \pm 0.19 \mathrm{~d}$ \\
\hline & \multirow{2}{*}{ Jiza127 } & NW & $26.72 \pm 1.20 \mathrm{a}$ & $0.17 \pm 0.01 \mathrm{a}$ & $2.77 \pm 0.05 \mathrm{a}$ \\
\hline & & WD & $20.52 \pm 1.33 b$ & $0.13 \pm 0.01 \mathrm{c}$ & $2.23 \pm 0.26 \mathrm{~cd}$ \\
\hline \multirow{8}{*}{ Anthesis } & \multirow{2}{*}{ Jiza305 } & NW & $25.67 \pm 0.64 \mathrm{a}$ & $0.17 \pm 0.02 \mathrm{a}$ & $2.70 \pm 0.16 \mathrm{a}$ \\
\hline & & WD & $20.30 \pm 1.31 \mathrm{~b}$ & $0.13 \pm 0.03 b c$ & $2.14 \pm 0.11 b$ \\
\hline & \multirow{2}{*}{ Jinza106 } & NW & $25.47 \pm 0.45 \mathrm{a}$ & $0.16 \pm 0.03 \mathrm{ab}$ & $2.66 \pm 0.06 \mathrm{a}$ \\
\hline & & WD & $19.30 \pm 2.62 b$ & $0.12 \pm 0.00 \mathrm{c}$ & $2.01 \pm 0.15 b c$ \\
\hline & \multirow{2}{*}{ Jinza103 } & NW & $25.63 \pm 1.46 \mathrm{a}$ & $0.17 \pm 0.01 \mathrm{a}$ & $2.73 \pm 0.14 \mathrm{a}$ \\
\hline & & WD & $18.10 \pm 0.96 b c$ & $0.12 \pm 0.01 \mathrm{c}$ & $1.96 \pm 0.14 b c$ \\
\hline & \multirow{2}{*}{ Jiza127 } & NW & $25.93 \pm 1.70 \mathrm{a}$ & $0.17 \pm 0.00 \mathrm{a}$ & $2.66 \pm 0.01 \mathrm{a}$ \\
\hline & & WD & $16.23 \pm 0.45 \mathrm{c}$ & $0.11 \pm 0.00 \mathrm{c}$ & $1.77 \pm 0.20 \mathrm{c}$ \\
\hline \multirow{8}{*}{ Filling stage } & \multirow{2}{*}{ Jiza305 } & NW & $25.13 \pm 0.75 \mathrm{a}$ & $0.18 \pm 0.03 \mathrm{a}$ & $2.76 \pm 0.15 \mathrm{a}$ \\
\hline & & WD & $18.72 \pm 1.44 b$ & $0.14 \pm 0.01 \mathrm{~b}$ & $2.09 \pm 0.18 b$ \\
\hline & \multirow{2}{*}{ Jinza106 } & NW & $25.63 \pm 0.74 \mathrm{a}$ & $0.17 \pm 0.02 \mathrm{a}$ & $2.70 \pm 0.11 \mathrm{a}$ \\
\hline & & WD & $18.04 \pm 2.35 b$ & $0.12 \pm 0.00 \mathrm{bc}$ & $1.91 \pm 0.19 b c$ \\
\hline & \multirow{2}{*}{ Jinza103 } & NW & $25.52 \pm 2.81 \mathrm{a}$ & $0.17 \pm 0.01 \mathrm{a}$ & $2.80 \pm 0.13 \mathrm{a}$ \\
\hline & & WD & $16.67 \pm 2.35 \mathrm{bc}$ & $0.11 \pm 0.01 \mathrm{c}$ & $1.78 \pm 0.13 \mathrm{c}$ \\
\hline & \multirow{2}{*}{ Jiza127 } & NW & $25.23 \pm 0.55 \mathrm{a}$ & $0.18 \pm 0.02 \mathrm{a}$ & $2.70 \pm 0.17 \mathrm{a}$ \\
\hline & & WD & $14.43 \pm 1.02 \mathrm{c}$ & $0.11 \pm 0.00 \mathrm{c}$ & $1.64 \pm 0.14 \mathrm{c}$ \\
\hline
\end{tabular}

Note. NW - normal watering, WD - water deficit; mean \pm SD followed by different letters are significantly different by the Duncan's $(p<0.05)$.

Table 3. Variations of osmotic adjustment parameters of sorghum varieties under drought at jointing stage, anthesis and filling stage, respectively

\begin{tabular}{|c|c|c|c|c|c|}
\hline Stage & Variety & Treatment & $\begin{array}{c}\text { Relative water } \\
\text { content } \\
\%\end{array}$ & $\begin{array}{l}\text { Proline content } \\
\qquad \mu \mathrm{g} \mathrm{g}^{-1} \mathrm{FW}\end{array}$ & $\begin{array}{c}\text { Soluble sugar content } \\
\%\end{array}$ \\
\hline 1 & 2 & 3 & 4 & 5 & 6 \\
\hline \multirow{8}{*}{ Jointing stage } & \multirow{2}{*}{ Jiza305 } & NW & $95.93 \pm 0.63 \mathrm{a}$ & $42.46 \pm 1.21 \mathrm{~d}$ & $12.36 \pm 0.49 \mathrm{c}$ \\
\hline & & WD & $92.68 \pm 0.93 \mathrm{c}$ & $76.96 \pm 1.70 \mathrm{a}$ & $16.19 \pm 0.70 \mathrm{a}$ \\
\hline & \multirow{2}{*}{ Jinza106 } & NW & $94.91 \pm 0.81 \mathrm{ab}$ & $39.40 \pm 1.04 \mathrm{e}$ & $12.30 \pm 0.66 \mathrm{c}$ \\
\hline & & WD & $91.66 \pm 0.81 \mathrm{c}$ & $69.61 \pm 0.47 \mathrm{bc}$ & $15.64 \pm 0.53 \mathrm{ab}$ \\
\hline & \multirow{2}{*}{ Jinza103 } & NW & $94.16 \pm 0.49 b$ & $42.05 \pm 2.86 \mathrm{~d}$ & $12.22 \pm 0.65 \mathrm{c}$ \\
\hline & & WD & $89.41 \pm 1.02 \mathrm{~d}$ & $68.27 \pm 1.67 \mathrm{c}$ & $14.85 \pm 0.62 b$ \\
\hline & \multirow{2}{*}{ Jiza127 } & NW & $94.38 \pm 0.62 b$ & $44.72 \pm 0.73 \mathrm{~d}$ & $12.27 \pm 0.39 \mathrm{c}$ \\
\hline & & WD & $89.69 \pm 1.23 \mathrm{~d}$ & $71.26 \pm 1.09 \mathrm{~b}$ & $14.64 \pm 0.89 \mathrm{~b}$ \\
\hline \multirow{8}{*}{ Anthesis } & \multirow{2}{*}{ Jiza305 } & NW & $95.55 \pm 0.53 \mathrm{a}$ & $52.01 \pm 1.37 \mathrm{e}$ & $13.75 \pm 0.91 \mathrm{~cd}$ \\
\hline & & WD & $92.51 \pm 0.58 \mathrm{c}$ & $97.27 \pm 1.93 \mathrm{a}$ & $18.19 \pm 0.56 \mathrm{a}$ \\
\hline & \multirow{2}{*}{ Jinza106 } & NW & $95.88 \pm 0.95 \mathrm{a}$ & $47.92 \pm 1.54 \mathrm{f}$ & $13.41 \pm 0.60 \mathrm{~d}$ \\
\hline & & WD & $91.76 \pm 0.32 \mathrm{c}$ & $83.93 \pm 1.96 \mathrm{c}$ & $16.51 \pm 1.00 \mathrm{~b}$ \\
\hline & \multirow{2}{*}{ Jinza103 } & NW & $93.85 \pm 1.08 b$ & $49.24 \pm 1.15 \mathrm{f}$ & $13.39 \pm 0.49 \mathrm{~d}$ \\
\hline & & WD & $88.99 \pm 0.68 \mathrm{~d}$ & $87.92 \pm 0.64 b$ & $16.29 \pm 0.43 b$ \\
\hline & \multirow{2}{*}{ Jiza127 } & NW & $94.56 \pm 0.93 \mathrm{ab}$ & $50.58 \pm 2.14$ ef & $12.72 \pm 1.03 \mathrm{~d}$ \\
\hline & & WD & $89.07 \pm 0.65 \mathrm{~d}$ & $79.40 \pm 0.67 \mathrm{~d}$ & $15.10 \pm 0.97 b c$ \\
\hline
\end{tabular}


Table 3 continued

\begin{tabular}{cccccc}
\hline 1 & 2 & 3 & 4 & 5 & 6 \\
\hline \multirow{2}{*}{ Jiza305 } & NW & $95.45 \pm 0.51 \mathrm{a}$ & $49.44 \pm 1.18 \mathrm{~d}$ & $13.78 \pm 0.91 \mathrm{bcd}$ \\
& & WD & $91.29 \pm 0.41 \mathrm{~b}$ & $90.26 \pm 0.36 \mathrm{a}$ & $17.76 \pm 1.00 \mathrm{a}$ \\
& \multirow{2}{*}{ Jinza106 } & NW & $95.43 \pm 0.89 \mathrm{a}$ & $47.14 \pm 0.90 \mathrm{~d}$ & $12.42 \pm 0.42 \mathrm{de}$ \\
& & WD & $90.92 \pm 0.30 \mathrm{~b}$ & $76.46 \pm 0.74 \mathrm{~b}$ & $15.02 \pm 0.31 \mathrm{~b}$ \\
& \multirow{2}{*}{ Jinza103 } & NW & $90.27 \pm 0.84 \mathrm{bc}$ & $45.37 \pm 5.13 \mathrm{~d}$ & $11.85 \pm 0.60 \mathrm{e}$ \\
& & WD & $85.16 \pm 0.26 \mathrm{~d}$ & $69.92 \pm 1.09 \mathrm{c}$ & $13.72 \pm 1.78 \mathrm{bcd}$ \\
& Jiza127 & NW & $89.50 \pm 0.47 \mathrm{c}$ & $45.00 \pm 5.08 \mathrm{~d}$ & $12.88 \pm 0.68 \mathrm{cde}$ \\
& & WD & $83.62 \pm 1.04 \mathrm{e}$ & $68.38 \pm 0.74 \mathrm{c}$ & $14.36 \pm 0.49 \mathrm{bc}$ \\
\hline
\end{tabular}

Note. NW - normal watering, WD - water deficit; FW - fresh weight; mean \pm SD followed by different letters are significantly different by the Duncan's $(p<0.05)$.

Table 4. Variations of activities of antioxidant enzymes of sorghum varieties under drought at jointing stage, anthesis and filling stage, respectively

\begin{tabular}{|c|c|c|c|c|c|}
\hline Stage & Variety & Treatment & $\begin{array}{c}\text { Activity of } \\
\text { superoxide dismutase } \\
\mathrm{U} \mathrm{g}^{-1} \mathrm{FW}\end{array}$ & $\begin{array}{l}\text { Activity of peroxidase } \\
\Delta \mathrm{OD}_{470} \mathrm{~g}^{-1} \mathrm{~min}^{-1} \mathrm{FW}\end{array}$ & $\begin{array}{l}\text { Activity of catalase } \\
\mathrm{mg} \mathrm{H}_{2} \mathrm{O}_{2} \mathrm{~g}^{-1} \mathrm{~min}^{-1} \mathrm{FW}\end{array}$ \\
\hline \multirow{8}{*}{ Jointing stage } & \multirow{2}{*}{ Jiza305 } & NW & $292.39 \pm 0.94 \mathrm{c}$ & $142.78 \pm 2.80 \mathrm{~d}$ & $15.72 \pm 0.04 \mathrm{~d}$ \\
\hline & & WD & $342.51 \pm 0.88 \mathrm{a}$ & $172.13 \pm 3.26 \mathrm{a}$ & $17.89 \pm 0.06 \mathrm{a}$ \\
\hline & \multirow{2}{*}{ Jinza106 } & NW & $292.38 \pm 0.78 \mathrm{c}$ & $141.56 \pm 1.83 \mathrm{~d}$ & $15.50 \pm 0.22 \mathrm{e}$ \\
\hline & & WD & $341.69 \pm 0.50 \mathrm{a}$ & $168.16 \pm 3.45 \mathrm{ab}$ & $17.29 \pm 0.09 \mathrm{~b}$ \\
\hline & \multirow{2}{*}{ Jinza103 } & NW & $292.37 \pm 0.20 \mathrm{c}$ & $142.15 \pm 3.67 \mathrm{~d}$ & $15.28 \pm 0.07 \mathrm{f}$ \\
\hline & & WD & $332.86 \pm 0.65 \mathrm{~b}$ & $162.91 \pm 3.01 \mathrm{bc}$ & $16.47 \pm 0.11 \mathrm{c}$ \\
\hline & \multirow{2}{*}{ Jiza127 } & NW & $292.37 \pm 0.75 \mathrm{c}$ & $142.13 \pm 3.59 \mathrm{~d}$ & $15.26 \pm 0.08 \mathrm{f}$ \\
\hline & & WD & $332.65 \pm 0.69 \mathrm{~b}$ & $161.38 \pm 2.14 \mathrm{c}$ & $16.48 \pm 0.05 \mathrm{c}$ \\
\hline \multirow{8}{*}{ Anthesis } & \multirow{2}{*}{ Jiza305 } & NW & $329.44 \pm 4.29 \mathrm{e}$ & $212.64 \pm 2.78 \mathrm{~d}$ & $15.17 \pm 0.02 \mathrm{e}$ \\
\hline & & WD & $408.67 \pm 3.78 \mathrm{a}$ & $258.91 \pm 2.77 \mathrm{a}$ & $17.44 \pm 0.11 \mathrm{a}$ \\
\hline & \multirow{2}{*}{ Jinza106 } & NW & $330.29 \pm 5.67 \mathrm{e}$ & $211.01 \pm 1.43 \mathrm{~d}$ & $14.81 \pm 0.02 \mathrm{f}$ \\
\hline & & WD & $388.50 \pm 1.01 \mathrm{bc}$ & $243.53 \pm 3.20 b$ & $16.58 \pm 0.01 \mathrm{~b}$ \\
\hline & \multirow{2}{*}{ Jinza103 } & NW & $329.00 \pm 3.83 \mathrm{e}$ & $210.13 \pm 1.76 \mathrm{~d}$ & $14.49 \pm 0.01 \mathrm{~g}$ \\
\hline & & WD & $391.98 \pm 2.98 b$ & $241.33 \pm 2.44 b$ & $16.16 \pm 0.03 \mathrm{c}$ \\
\hline & \multirow{2}{*}{ Jiza127 } & NW & $339.03 \pm 2.95 \mathrm{~d}$ & $209.51 \pm 1.42 \mathrm{~d}$ & $14.36 \pm 0.01 \mathrm{~h}$ \\
\hline & & WD & $385.29 \pm 1.10 \mathrm{c}$ & $231.32 \pm 1.75 \mathrm{c}$ & $15.74 \pm 0.01 \mathrm{~d}$ \\
\hline \multirow{8}{*}{ Filling stage } & \multirow{2}{*}{ Jiza305 } & NW & $323.26 \pm 5.44 \mathrm{e}$ & $208.57 \pm 2.57 \mathrm{e}$ & $10.31 \pm 0.08 \mathrm{c}$ \\
\hline & & WD & $401.23 \pm 7.84 \mathrm{a}$ & $249.10 \pm 0.71 \mathrm{a}$ & $11.59 \pm 0.08 \mathrm{a}$ \\
\hline & \multirow{2}{*}{ Jinza106 } & NW & $319.72 \pm 1.65 \mathrm{e}$ & $207.17 \pm 0.95$ ef & $9.59 \pm 0.04 \mathrm{~d}$ \\
\hline & & WD & $374.43 \pm 4.80 \mathrm{~b}$ & $238.59 \pm 1.48 \mathrm{~b}$ & $10.51 \pm 0.02 \mathrm{~b}$ \\
\hline & \multirow{2}{*}{ Jinza103 } & NW & $319.23 \pm 2.47 \mathrm{e}$ & $205.68 \pm 2.99$ ef & $7.16 \pm 0.04 \mathrm{~g}$ \\
\hline & & WD & $357.48 \pm 0.55 \mathrm{c}$ & $233.97 \pm 1.01 \mathrm{c}$ & $7.70 \pm 0.04 \mathrm{e}$ \\
\hline & \multirow{2}{*}{ Jiza127 } & NW & $333.79 \pm 2.22 \mathrm{~d}$ & $204.30 \pm 2.67 \mathrm{f}$ & $7.13 \pm 0.00 \mathrm{~g}$ \\
\hline & & WD & $369.22 \pm 5.38 \mathrm{~b}$ & $224.12 \pm 1.90 \mathrm{~d}$ & $7.59 \pm 0.02 \mathrm{f}$ \\
\hline
\end{tabular}

Note. NW - normal watering, WD - water deficit; FW - fresh weight; mean \pm SD followed by different letters are significantly different by the Duncan's $(p<0.05)$.

In our study, the nine physiological parameters investigated in the present study can be divided into three categories according to their function, i.e. gas-exchange parameters, osmotic adjustment, and antioxidant metabolism. Our results showed that the gas-exchange parameters, including transpiration rate, stomatal conductance and photosynthetic rate, were the primary factors that influenced drought tolerance of sorghum (Table 5). The secondary parameters were related to osmotic adjustment, including soluble sugar content, proline content and relative water content. The tertiary parameters were related to antioxidant metabolism, including activities of CAT, SOD and POD. These results were partly supported by Wang et al. (2007) who conducted a grey relational analysis in wheat under natural drought conditions and found that stomatal conductance had the closest relationship with the drought tolerance. 
Table 5. Grey relational degree of all investigated physiological parameters

\begin{tabular}{lcc}
\hline \multicolumn{1}{c}{ Physiological parameters } & $\begin{array}{c}\text { Grey relational } \\
\text { degree }\end{array}$ & Rank \\
\hline Transpiration rate & 0.8111 & 1 \\
Stomatal conductance & 0.7759 & 2 \\
Photosynthetic rate & 0.7517 & 3 \\
Soluble sugar content & 0.7075 & 4 \\
Proline content & 0.7048 & 5 \\
Relative water content & 0.6873 & 6 \\
Activity of catalase & 0.6835 & 7 \\
Activity of superoxide dismutase & 0.6549 & 8 \\
Activity of peroxidase & 0.6537 & 9 \\
\hline
\end{tabular}

Gas exchange parameters are determined by the movement of stoma which is an important channel for plants to exchange the moisture and gas. Stomata opening may decrease or even close when plants lose moisture under drought stress, which is significant for plants to survive under drought conditions. However, the decreased degree of stomatal opening also prevents $\mathrm{CO}_{2}$ from entering plants through stomata. In this study, the inhibition of photosynthetic rate among sorghum varieties as a result of drought stress resulted from the decrease of stomatal conductance (Table 2). Zhang et al. (2011) suggested that a decrease in $\mathrm{CO}_{2}$ assimilation due to the closure of stomata may lead to a damage of photosystem II because of excess energy. The ultimate purpose of crop production is to obtain relative high yield, and especially under drought conditions the artificial selection of drought-tolerant germplasm for yield would need to satisfy photosynthesis with adequate carbon dioxide concentration to maintain assimilative capacity. Furthermore, these gas-exchange parameters are often referred to photosynthesis. Photosynthesis is the basis of crop yield formation. As to guarantee yield formation, it is important to maintain photosynthetic ability under drought stress. From our results, drought stress at filling stage had the greatest effect on photosynthesis and the photosynthetic performance of sorghum varieties was consistent with their final yield, respectively.

Increasing contents of osmotic adjustment substances can improve the hydrophilicity of the cell and reduce water loss under drought conditions. In the study, the ability of osmotic adjustment of sorghum varieties was motivated under drought conditions by increasing the contents of proline and soluble sugar (Table 3 ). In addition, the increased extents of osmotic adjustment substances induced by drought were generally higher than the increased extents of activities of antioxidant enzymes at the same stage, suggesting osmotic adjustment played more important role in drought tolerance. Previous studies documented that exogenous osmotic adjustment substances could enhance activities of antioxidant enzymes (Nounjan et al., 2012; Hou et al., 2013; Huang et al., 2013). Malondialdehyde is unavoidably produced when polyunsaturated fatty acids in the membrane undergo peroxidation due to the adverse conditions and it is often used as an indicator of plant injury. It is more important to preserve water status to maintain normal metabolization in crops under drought, although activities of antioxidant enzymes are often increased to inhibit peroxidation during drought stresses.

\section{Conclusions}

1. Drought tolerance index of yield (DTIY) was a reliable indicator to reflect sorghum performance under drought stress. The grey relational analysis (GRA) could be applied as an effective way for sorghum breeders to compare the importance of physiological parameters to improve the efficiency and accuracy of selection of drought tolerance for the germplasm evaluation and breeding in sorghum.

2. Gas exchange parameters were the principal factors in evaluating the drought tolerance associated with sorghum yield formation under drought conditions. Osmotic adjustment ability was more important as compared to the activities of antioxidant enzymes when sorghum suffered from drought.

\section{Acknowledgements}

This work was funded by the sub-project of the National Science and Technology Support Program (2014BAD07B02) of Chinese Ministry of Science and Technology, and China Agriculture Research System (CARS-06-02-02), as well as Postdoctoral Fund of Shenyang Agricultural University.

Received 27062015

Accepted 22092015

\section{References}

Assefa T., Beebe S. E., Rao I. M., Cuasquer J. B., Duque M. C., Rivera M., Battisti A., Lucchin M. 2013. Pod harvest index as a selection criterion to improve drought resistance in white pea bean. Field Crops Research, 148: 24-33 http://dx.doi.org/10.1016/j.fcr.2013.04.008

Borrell A. K., van Oosterom E. J., Mullet J. E., George-Jaeggli B., Jordan D. R., Klein P. E., Hammer G. L. 2014. Stay-green alleles individually enhance grain yield in sorghum under drought by modifying canopy development and water uptake patterns. New Phytologist, 203: 817-830 http://dx.doi.org/10.1111/nph.12869

Chen X. J., Min D. H., Yasir T. A., Hua Y. G. 2012. Evaluation of 14 morphological, yield-related and physiological traits as indicators of drought tolerance in Chinese winter bread wheat revealed by analysis of the membership function value of drought tolerance (MFVD). Field Crops Research, 137: 195-201 http://dx.doi.org/10.1016/j.fcr.2012.09.008

Deng J. L. 1989. Introduction to grey system theory. Journal of Grey System, 1: 1-24

FAO. 2015. Food and Agriculture Organization of the United Nations. $<$ http://faostat3.fao.org/download/Q/QC/E> 
Hou P. F., Ma J. Q., Zhao P. F., Zhang H. L., Zhao H. J., Liu H. Sh., Zhao Y. D., Wang Y. X. 2013. Effects of betaine on chloroplast protective enzymes and $p s b A$ gene expression in wheat seedlings under drought stress. Acta Agronomica Sinica, 39 (7): 1319-1324 http://dx.doi.org/10.3724/SP.J.1006.2013.01319

Hu G., Zhang J., Fan L. 2012. Influential factors of water productivity of maize in oasis of arid areas - a case study of linze county. Agricultural Science and Technology, 13 (9): 1854-4858

Huang Y. W., Nie Y. X., Wan Y. Y., Chen S. Y., Sun Y., Wang X. J., Bai J. G. 2013. Exogenous glucose regulates activities of antioxidant enzyme, soluble acid invertase and neutral invertase and alleviates dehydration stress of cucumber seedlings. Scientia Horticulturae, 162: 20-30 http://dx.doi.org/10.1016/j.scienta.2013.07.031

Jia Y. H., Shao M. A. 2013. Temporal stability of soil water storage under four types of revegetation on the northern Loess Plateau of China. Agricultural Water Management, 117: $33-42$ http://dx.doi.org/10.1016/j.agwat.2012.10.013

Lan J. S., Hu F. S., Zhang J. R. 1990. The concept and statistical method of drought resistance index in crop. Acta Agriculturae Boreali Sinica, 5 (2): 20-25

Li H. Sh. 2000. The principle and technology of plant physiology and biochemistry experiment. Beijing, China, 195-196 p.

Li Y. Y., Feng Z., Zhao L. Y., Mo Z. H., Zhang B. 2007. The grey analysis, kriging and selection index of flower yield in rugosa rose. Agricultural Sciences in China, 6 (12): $1420-1425$ http://dx.doi.org/10.1016/S1671-2927(08)60003-6

Lu Y. L., Hao Z. F., Xie C. X., Crossa J., Araus J. L., Gao S. B., Vivek B. S., Magorokosho C., Mugo S., Makumbi D., Taba S., Pan G. T., Li X. H., Rong T. Z., Zhang S. H., Xu Y. B. 2011. Large-scale screening for maize drought resistance using multiple selection criteria evaluated under water-stressed and well-watered environments. Field Crops Research, 124: 37-45 http://dx.doi.org/10.1016/j.fcr.2011.06.003

Nounjan N., Nghia P. T., Theerakulpisut P. 2012. Exogenous proline and trehalose promote recovery of rice seedlings from salt-stress and differentially modulate antioxidant enzymes and expression of related genes. Journal of Plant Physiology, 169: 596-604 http://dx.doi.org/10.1016/j.jplph.2012.01.004

Ober E. S., Le Bloa M., Clark C. J. A., Royal A., Jaggard K. W., Pidgeon J. D. 2005. Evaluation of physiological traits as indirect selection criteria for drought tolerance in sugar beet. Field Crops Research, 91: 231-249 http://dx.doi.org/10.1016/j.fcr.2004.07.012

Ogbaga C. C., Stepien P., Johnson G. N. 2014. Sorghum (Sorghum bicolor) varieties adopt strongly contrasting strategies in response to drought. Physiologia Plantarum, 152: $389-401$ http://dx.doi.org/10.1111/ppl.12196

Shan L., Xu B. C. 2009. Discussion on drought resistance of sorghum and its status in agriculture in arid and semiarid regions. Scientia Agricultura Sinica, 42 (7): 2342-2348

Wang H. F., Guo W., Wang J. H., Huang W. J., Gu X. H., Dong Y. Y., Xu X. G. 2013. Exploring the feasibility of winter wheat freeze injury by integrating grey system model with RS and GIS. Journal of Integrative Agriculture, 12 (7): 1162-1172

http://dx.doi.org/10.1016/S2095-3119(13)60445-1
Wang S. Q., Hu Y. G., She K. J., Zhou L. L., Meng F. L. 2007. Gray relational grade analysis of agronomical and physicbiochemical traits related to drought tolerance in wheat. Scientia Agricultura Sinica, 40 (11): 2452-2459

Zhang R. H., Zheng Y. J., Ma G. S., Zhang X. H., Lu H. D., Shi J. T., Xue J. Q. 2011. Effects of drought stress on photosynthetic traits and protective enzyme activity in maize seedling. Acta Ecologica Sinica, 31: 1303-1311

Zhang X. Z. 1992. Crop physiology research methods. Beijing, China, 208-212 p.

Zhou Y. F., Wang D. Q., Lu Z. B., Wang N., Wang Y. T., Li F. X., Xu W. J., Huang R. D. 2013. Effect of drought stress on osmotic adjustment and chloroplast ultrastructure of staygreen sorghum. Chinese Journal of Applied Ecology, 24 (9): 2545-2550

Zhou Y. F., Wang D. Q., Lu Z. B., Wang N., Wang Y. T., Li F. X., Xu W. J., Huang R. D. 2014. Effects of drought stress on photosynthetic characteristics and endogenous hormone ABA and CTK contents in stay-green sorghum. Scientia Agricultura Sinica, 47 (4): 655-663 


\title{
Galimų fiziologinių rodiklių prioritizavimas vertinant sorgų atsparumą sausrai: pilkoji reliacinè analizė
}

\author{
N. Wang ${ }^{1}$, Y. Wang ${ }^{2}$, J. Yu $u^{3}$, Y. Zhou ${ }^{1}$, Q. Wu ${ }^{1}$, Y. Gao ${ }^{1}$, W. Xu' ${ }^{1}$, R. Huang ${ }^{1}$ \\ ${ }^{1}$ Shenyang žemės ūkio universiteto Agronomijos koledžas, Kinija \\ ${ }^{2}$ Kinijos agroaplinkos apsaugos ir priežiūros centras \\ ${ }^{3}$ Džordžijos universitetas, JAV
}

\section{Santrauka}

Dvispalvị sorgą (Sorghum bicolor L. Moench) selekcionuojant ir auginant sausros sąlygomis yra svarbu nustatyti ir įvertinti sausrai atsparią genetinę medžiagą. Tyrimo tikslas - taikant pilkają reliacinę analizę ištirti galimų fiziologinių rodiklių reikšmę vertinant sorgų atsparumą sausrai. Keturių veislių sorgai buvo auginti vegetaciniuose induose bamblëjimo, žydèjimo ir grūdo pildymosi tarpsniais taikant du drèkinimo variantus - normalų drèkinimą (75-80\% dirvos drègmès imlumo) ir drègmès deficitą (45-50\% dirvos drègmès imlumo). Vertinant sorgų augimą sausros sąlygomis kaip pagrindinis rodiklis buvo naudojamas derliaus atsparumo sausrai indeksas. Tirtų rodiklių pilkasis reliacinis laipsnis mažèjo taip: transpiracijos greitis $>$ žiotelių laidumas $>$ tirpaus cukraus kiekis $>$ prolino kiekis $>$ santykinis drègmès kiekis $>$ katalazès veikla $>$ peroksidazès superoksidazès dismutazès aktyvumas. Tai reiškia, kad atsparumas sausrai, užtikrinantis sorgų derliaus formavimąsi, buvo labiausiai susijęs su dujų apykaitos rodikliais. Veikiant sausros stresui labai svarbus augalų augimo rodiklis buvo drègmès kiekis, palyginus su antioksidacinių fermentų aktyvumu.

Tyrimo rezultatai parodè, kad tirti rodikliai gali būti naudojami vertinant sorgų atsparumą sausrai, siekiant pagerinti atrankos efektyvumą ir tikslumą.

Reikšminiai žodžiai: analitinis metodas, drègmès deficitas, fiziologiniai požymiai, Sorghum bicolor. 